\title{
THE SINGULAR MEASURE OF A DIRICHLET SPACE
}

\author{
MASAYUKI ITÔ
}

\section{Introduction}

We [4], [5] examined some properties of balayaged measures in the theory of a Dirichlet space. In those papers, we showed that the singular measure of a Dirichlet space plays some important roles. In this paper, we shall precisely examine some properties of the singular measure of a Dirichlet space. Let $X$ be a locally compact Hausdorff space in which there exists a positive Radon measure $\xi$ which is everywhere dense in $X$. First we obtain the following

(1) Let $D$ be a Dirichlet space with respect to $X$ and $\xi$, and let $\sigma$ be the singular measure of $D$. For any couple $u$ and $v$ in $D$ such that $S_{u} \cap S_{v}=\phi,{ }^{1)}$ the function $u^{*}(x) v^{*}(y)$ in the product space $X \times X$ is $\sigma$ integrable and

$$
(u, v)=-2 \iint u^{*}(x) v^{*}(y) d \sigma(x, y),
$$

where $u^{*}$ and $v^{*}$ are the refinements of $u$ and $v$, respectively.

By using this result, we shall obtain more precise results than those in [4]. Moreover we have the following

(2) Let $D$ be the same as the above (1), and let $u_{\mu}$ be a pure potential in $D$. For an open set $\omega$ in $X$, let $\mu^{\prime}$ be the balayaged measure of $\mu$ to $\omega$, and let $\nu^{\prime}$ be the restriction of $\mu^{\prime}$ to $\omega$. For any pure potential $u_{\mu}$ in $D$ and any open set $\omega$ contained in the complement $C S_{\mu}$ of the support of $\mu, \nu^{\prime}$ is absolutely continuous for $\xi$ if and only if the projection of the singular measure of $D$ to $X$ is absolutely continuous for $\xi$.

Next we shall examine total masses of balayaged measures. The result in this paper is better than the one in [5].

Finally we shall obtain more precise results in the case of a special Dirichlet space. Especially the following result is important.

Received February 17, 1967.

1) For a $\xi$-measurable function $f, S_{f}$ means the complement of the largest open set $\omega$ such that $f(x)=0$ in $\omega$. 
For any special Dirichlet space $D, \nu^{\prime}$ is always absolutely continuous for $\xi$.

\section{Preliminaries on Dirichlet spaces}

Let $X$ be a locally compact Hausdorff space in which there exists a positive Radon measure $\xi$ which is everywhere dense in $X$ (i.e., $\xi(\omega)>0$ for any non-empty open set $\omega$ in $X$ ). Let $C_{K}$ be the space of finite continuous functions with compact support provided with the topology usual. According to Beurling \& Deny [2], we define a $\xi$-Dirichlet space on $X$.

Definition 1. A Hilbert space $D=D(X ; \xi)$ is called $\xi$-Dirichlet space (simply, Dirichlet space) on $X$ if each element $u$ in $D$ is locally $\xi$-summable (simply, summable) real-valued function ${ }^{2}$ ) in $X$ and the following three conditions are satisfied:

(D. 1) For any compact subset $K$ of $X$, there exists a positive constant $A(K)$ such that

$$
\int_{K}|u(x)| d \xi(x) \leq A(K)\|u\|
$$

for any $u$ in $D$.

(D. 2) $C_{K} \cap D$ is dense both in $C_{K}$ and in $D$.

(D. 3) For any $u$ in $D$ and any normal contraction $T$ on the real line $R$, $T \cdot u$ is contained in $D$ and $\|T \cdot u\| \leq\|u\|$.

In the above (D. 3), A transformation $T$ on $R$ into itself is called a normal contraction if it satisfies the following:

$$
T(0)=0 \text { and }\left|T a_{1}-T a_{2}\right| \leq\left|a_{1}-a_{2}\right|
$$

for any couple $a_{1}$ and $a_{2}$ in $R$. Two functions which are equal locally almost everywhere (simply, a.e.) for $\xi$ represents the same element in $D$. The norm of $D$ is denoted by $\|u\|$, the associated scalar product by $(u, v)$. Similarly as Beurling and Deny [2], we define potentials in $D$.

2) Beurling \& Deny [2] first assumed that each element $u$ in $D$ is a complex-valued function in $X$. Put $D_{r}=\{\operatorname{Re} u ; u \in D\}$. Then $D_{r}$ is a Dirichlet space in our sense. Conversely, let $D$ be a Dirichlet space in our sense. Put $D^{\prime}=\{u+i v ; u, v \in D\}$. Then $D^{\prime}$ is a Dirichlet space in Beurling \& Deny's sense. In potential theory, it is sufficient to assume that each $u$ in $D$ is real-valued, because important potentials, i.e., balayaged potentials, equilibrium potentials, $\cdots$ are all real-valued. 
Definition 2. An element $u$ in $D$ is called a potential in $D$ if there exists a real Radon measure $\mu$ in $X$ such that

$$
(f, u)=\int f(x) d \mu(x)
$$

for any $f$ in $C_{K} \cap D$. Such an element $u$ is denoted by $u_{\mu}$. Especially if $\mu$ is positive, $u_{\mu}$ is called a pure potential in $D$. By Definition 1, (D. 1), for each bounded measurable function $f$ with compact support, there exists a unique element $u_{f}$ in $D$ such that

$$
\left(v, u_{f}\right)=\int v(x) f(x) d \xi(x)
$$

for any $v$ in $D$.

Beurling and Deny [2] showed the following important representation theorem.

Proposition 1. For a Dirichlet space $D$ on $X$, there exist a positive measure $\nu$ in $X$, a positive Hermitian form $N(f, g)$ on $C_{K} \cap D$ and a positive symmetric measure $\sigma$ in $X \times X-\delta$ ( $\delta$ is the diagonal set of $X \times X)$ such that

$$
(f, g)=\int f g d \nu+N(f, g)+\iint(f(x)-f(y))(g(x)-g(y)) d \sigma(x, y)
$$

for any couple $f$ and $g$ in $C_{K} \cap D$. Here $N(f, g)$ has the following local character: if $g$ is constant in some neighborhood of the support $S_{f}$ of $f$, then $N(f, g)$ vanishes.

Proposition 2. For a Dirichlet space $D$ on $X$, the above representation is unique.

Proof. Suppose that there exist another positive measure $\nu^{\prime}$ in $X$, another positive Hermitian form $N^{\prime}(f, g)$ on $C_{K} \cap D$ with the above local character and another positive symmetric measure $\sigma^{\prime}$ in $X \times X-\delta$ such that

$$
(f, g)=\int f g d \nu+N^{\prime}(f, g)+\iint(f(x)-f(y))(g(x)-g(y)) d \sigma^{\prime}(x, y)
$$

for any couple $f$ and $g$ in $C_{K} \cap D$. Since $C_{K} \cap D$ is dense in $C_{K}$, the set

$$
\left\{f(x) g(y) ; f, g \in C_{K} \cap D, S_{f} \cap S_{g}=\phi\right\}
$$

is dense in $C_{K}(X \times X-\delta) .^{3)} \quad$ For any couple $f$ and $g$ in $C_{K} \cap D$ with $S_{f} \cap S_{g}=\phi$,

3) $C_{K}(X \times X-\delta)$ is the space of finite continuous functions in $X \times X-\delta$ with compact support provided with the topology of uniform convergence. 


$$
(f, g)=-2 \int f(x) g(y) d \sigma(x, y)=-2 \int f(x) g(y) d \sigma^{\prime}(x, y)
$$

Hence the equality $\sigma=\sigma^{\prime}$ holds. Next we shall show the equality $\nu=\nu^{\prime}$. It is sufficient to prove the equality

$$
\int f d \nu=\int f d \nu^{\prime}
$$

for any $f$ in $C_{K} \cap D$. Similarly as in the proof of Theorem 1 in [4], there exists a function $g$ in $C_{K} \cap D$ such that $g(x)=1$ in some neighborhood of $S_{f}$. The Hermitian forms $N(f, g)$ and $N^{\prime}(f, g)$ having the local character,

$$
\begin{gathered}
\quad(f, g)=\int f d \nu+\iint(f(x)-f(y))(g(x)-g(y)) d \sigma(x, y) \\
=\int f d \nu^{\prime}+\iint(f(x)-f(y))(g(x)-g(y)) d \sigma^{\prime}(x, y) .
\end{gathered}
$$

Therefore the equality $\nu=\nu^{\prime}$ holds, and hence

$$
N(f, g)=N^{\prime}(f, g)
$$

on $C_{K} \cap D$. This completes the proof.

Definition 3. The above measure $\nu$ in $X$ is called the equilibrium measure of $X$ (with respect to $D),{ }^{4)} N(f, g)$ is called the local form of $D$ and the positive measure $\sigma$ is called the singular measure of $D$.

\section{Some lemmas}

In order to obtain our first main theorem, we need the following lemmas.

Lemma 1. Let $D$ be a Dirichlet space on $X$. For a compact set $F_{1}$ and a closed set $F_{0}$ in $X$ with $F_{1} \cap F_{0}=\phi$, let $u_{\mu_{1}-\mu_{0}}$ be the condensor potential with respect to $F_{1}$ and $\left.F_{0 .}{ }^{5}\right)$ Then $u_{\mu_{1}-\mu_{0}}$ is contained in the closure of the following subset $E_{1,0}$ of $D$ :

$$
E_{1,0}=\left\{f \in C_{K} \cap D ; f(x)=1 \text { on } F_{1} \text { and } f(x)=0 \text { on } F_{0}\right\} .
$$

4) Beurling and Deny [2] remarked that for any non-decreasing net $\left(\omega_{\alpha}\right)_{\alpha \in I}$ of relatively compact open sets tending to $X$, the equilibrium measure of $\omega_{\alpha}$ tends vaguely to $\nu$. Hence we say that $\nu$ is the equilibrium measure of $X$.

5) Beurling and Deny [2] showed that for any couple of open sets $\omega_{1}$ and $\omega_{0}$ in $X, \omega_{1}$ being relatively compact, there exists a potential $u_{\mu_{1}-\mu_{0}}$ in $D$ satisfying the following: $0 \leq u_{\mu_{1}-\mu_{0}} \leq 1, u_{\mu_{1}-\mu_{0}}(x)=i$ a.e. in $\omega_{i}$ and $\mu_{i}$ is a positive measure in $X$ supported by $\overline{\omega_{i}}$. We [6] formed a similar potential in $D$ for a compact set $F_{1}$ and a closed set $F_{0}$. This potential is called the condensor potential with respect to $\omega_{1}$ and $\omega_{0}$ (or $F_{1}$ and $F_{0}$ ). 
Proof. We put

$$
\widetilde{E}_{1,0}=\left\{f \in C_{K} \cap D ; f(x) \geq 1 \text { on } F_{1} \text { and } f(x) \leq 0 \text { on } F_{0}\right\} .
$$

Then $\tilde{E}_{1,0}$ is a closed convex set and non-empty, because $C_{K} \cap D$ is dense in $C_{K}$. Let $u_{1,0}$ be a unique element in $\tilde{E}_{1,0}$ whose norm is minimal in $\widetilde{E}_{1,0}$. Similarly as Beurling and Deny's Condensor Theorem, we obtain that $u_{1,0}$ is equal to a potential $u_{\mu}$ in $D$ and $\mu^{+}$(resp. $\mu^{-}$) is supported by $F_{1}$ (resp. $F_{0}$ ). By the condition (D. 3) in Definition 1, $0 \leq u_{1,0} \leq 1$ and $u_{1,0}^{*}(x)=i p p p$ on $F_{i}$ for $\left.i=1,0,6\right)$ where $u_{1,0}^{*}$ is the refinement of $u_{1,0.7}$ ) Next we shall show that $u_{\mu_{1}-\mu_{0}}=u_{1,0}$. By Beurling and Deny's theorem, ${ }^{8)}$ there exists a sequence $\left(u_{\mu_{n}}\right)$ of linear combinations of pure potentials in $D$ such that $\left(u_{\mu_{n}}\right)$ converges strongly to $u_{1,0}$ as $n \longrightarrow+\infty$ and

$$
S_{\mu_{n}} \subset F_{1} \cup F_{0}
$$

Then we have

$$
\begin{aligned}
& \left\|u_{1,0}\right\|^{2}=\lim _{n \rightarrow \infty}\left(u_{1,0}, u_{\mu_{n}}\right)=\lim _{n \rightarrow \infty}\left(u_{\mu_{1}-\mu_{0}}, u_{\mu_{n}}\right) \\
= & \left(u_{\mu_{1}-\mu_{0}}, u_{1,0}\right) \leq\left\|u_{\mu_{1}-\mu_{0}}\right\| \cdot\left\|u_{1,0}\right\|,
\end{aligned}
$$

because

$$
u_{\mu_{1}-\mu_{0}}^{*}(x)=1 p p p \text { on } F_{1} \text { and } u_{\mu_{1}-\mu_{0}}^{*}(x)=0 p p p \text { on } F_{0} \text {. }
$$

That is,

$$
\left\|u_{1,0}\right\| \leq\left\|u_{\mu_{1}-\mu_{0}}\right\| \text {. }
$$

By the definition of the condensor potential, we obtain that $u_{1,0}=u_{\mu_{1}-\mu_{0}}$. Finally we shall prove that $u_{1,0} \in \overline{E_{1,0}}$. By the above assertion, there exists a sequence $\left(f_{n}^{\prime}\right)$ in $E_{1,0} \cap C_{K}$ such that $\left(f_{n}^{\prime}\right)$ converges strongly to $u_{1,0}$ in $D$. Let $T$ be the unit contraction on $R,{ }^{9}$ ) and put

$$
f_{n}(x)=T \cdot f_{n}^{\prime}(x) \text {. }
$$

6) A property is said to hold $p p p$ on a subset $E$ in $X$ if the property holds $\mu$-a.e. on $E$ for any pure potential $u_{\mu}$ in $D$ such that $S_{\mu} \subset E$.

7) Cf. [2], pp. 209-210.

8) Cf. [2], p. 214.

9) We say that the projection on $R$ to the closed interval $[0,1]$ is the unit contraction on R. Cf. [6]. 
Then $f_{n}$ is contained in $E_{1,0}$ and $\left(f_{n}\right)$ converges strongly to $u_{1,0}$ in $D$ as $n \longrightarrow+\infty$, because $\left(\left\|f_{n}\right\|\right)$ is bounded and

$$
\left\|u_{1,0}\right\|=\lim _{n \rightarrow \infty}\left\|f_{n}^{\prime}\right\| \geq \varlimsup_{n \rightarrow \infty}\left\|f_{n}\right\|
$$

This completes the proof.

Similarly as in the case of a special Dirichlet space, we obtain the following

Lemma 2. Let $D$ be a Dirichlet space on $X$ and $\sigma$ be the singular measure of D. For any compact set $K$ in $X$ and any open neighborhood $\omega$ of $K$,

$$
\iint_{K} d \sigma(x, y)<+\infty
$$

Proof. We take another open neighborhood $\omega^{\prime}$ of $K$ such that $\overline{\omega^{\prime}} \subset \omega$. Let $u_{\mu}$ be the condensor potential with respect to $K$ and $C \omega^{\prime}$ and let $\left(f_{n}\right)$ be a sequence in $C_{K} \cap D$ such that $\left(f_{n}\right)$ converges strongly to $u_{\mu}$ in $D$ as $n \longrightarrow+\infty$ and

$$
0 \leq f_{n}(x) \leq 1, f_{n}(x)=1 \text { on } K \text { and } f_{n}(x)=0 \text { on } C \omega^{\prime} \text {. }
$$

Let $\left(K_{\alpha}^{\prime}\right)_{\alpha \in I}$ be a non-decreasing net of compact subsets in $X$ tending to $X$ and put

$$
K_{\alpha}=K_{\alpha}^{\prime} \cap C \omega .
$$

Similarly as above, we can take a non-decreasing net $\left(g_{\alpha}\right)$ in $C_{K} \cap D$ such that

$$
S_{g_{\alpha}} \subset C \overline{\omega^{\prime}}, 0 \leq g_{\alpha} \leq 1 \text { and } g_{\alpha}(x)=1 \text { on } K_{\alpha} \text {. }
$$

Then for any $n$,

$$
\begin{aligned}
& \iint_{K \times K_{\alpha}} d \sigma(x, y) \leq \iint f_{n}(x) g_{\alpha}(y) d \sigma(x, y) \\
= & -\frac{1}{2}\left(f_{n}, g_{\alpha}\right) .
\end{aligned}
$$

Consequently we have 


$$
\iint_{K \times K_{\alpha}} d \sigma(x, y) \leq-\frac{1}{2}\left(u_{\mu}, g_{\alpha}\right)=\frac{1}{2} \int g_{\alpha}(x) d \mu^{-}(x) .
$$

The total mass of the positive measure $\mu^{-}$being finite, we obtain that

$$
\iint_{K \times C \omega} d \sigma(x, y) \leq \frac{1}{2} \int d \mu^{-}<+\infty .
$$

This completes the proof.

\section{First main theorem}

Now we define the projection of a singular measure of a Dirichlet space.

Definition 4. Let $\sigma$ be the singular measure of a Dirichlet space $D$. For a compact set $K$ in $X$, the projection $\sigma_{K}$ of $\sigma$ to $C K$ is the positive measure in $C K$ defined as follows:

$$
\int f d \sigma_{K}=\int_{K} \int f(y) d \sigma(x, y)
$$

for any $f$ in $C_{K}(C K)$.

Lemma 3. Let $\sigma$ be the singular measure of a Dirichlet space $D$. For a compact set $K$ in $X$ and an element $u$ in $D$ such that $K \cap S_{u}=\phi$, the refinement $u^{*}$ of $u$ is $\sigma_{K}$-integrable.

Proof. It is sufficient to prove that there exists a pure potential $u_{\mu}$ in $D$ such that the inequality $\sigma_{K} \leq \mu$ holds in an open set $\omega$ contained with its closure in $C K$. We take a couple of open sets $\omega_{1}$ and $\omega_{0}$ with disjoint closures, $\omega_{1}$ being relatively compact and holding the following inclusions:

$$
\omega_{1} \supset K \text { and } \omega_{0} \supset \bar{\omega} \text {. }
$$

Let $u_{\mu_{1}-\mu_{0}}$ be the condensor potential with respect to $\omega_{1}$ and $\omega_{0}$. Then by the results in the preceding paper, ${ }^{10)} u_{\mu_{1}}$ and $u_{\mu_{0}}$ are elements in $D$. Similarly as the above lemmas, there exists a sequence $\left(f_{n}\right)$ in $C_{K} \cap D$ such that $\left(f_{n}\right)$ converges strongly to $u_{\mu_{1}-\mu_{0}}$ as $n \longrightarrow+\infty$,

$$
0 \leq f_{n} \leq 1, f_{n}(x)=1 \text { on } K \text { and } f_{n}(x)=0 \text { on } \bar{\omega} .
$$

For any $f$ in $C_{K}^{+} \cap D^{11)}$ with support in $\omega$, we have

10) Cf. Levy-Khinchine's theorem in [2] and [3].

11) Cf. Lemma 1 and Lemma 3 in [5]. 


$$
\int_{K} \int f(y) d \sigma(x, y) \leq \iint f_{n}(x) f(y) d \sigma(x, y)=-\frac{1}{2}\left(f, f_{n}\right)
$$

for any $n$. Making $n$ tend to infinity, we obtain that

$$
\int_{K} \int f(y) d \sigma(x, y) \leq-\frac{1}{2}\left(f, u_{\mu_{1}-\mu_{0}}\right)=\frac{1}{2} \int f d \mu_{0} .
$$

$C_{K} \cap D$ being dense in $C_{K}$, we obtain that $\sigma_{K} \leq \frac{1}{2} \mu_{0}$ in $\omega$. This completes the proof.

By the above lemma, we obtain the following

Theorem 1. Let $D$ be a Dirichlet space on $X$ and $\sigma$ be the singular measure of $D$. For any potential $u_{\mu}$ in $D$, let $\mu^{(1)}$ be the restriction of $\mu$ to $C S_{u_{\mu}}$. Then

$$
d \mu^{(1)}(x)=-\frac{1}{2} \int u_{\mu}^{*}(y) d \sigma(x, y)
$$

in $C S_{u_{\mu}}$. Furthermore for any couple of elements $u_{1}$ and $u_{2}$ in $D$ such that $S_{u_{1}} \cap S_{u_{2}}=\phi$, we obtain

$$
\left(u_{1}, u_{2}\right)=-2 \iint u_{1}^{*}(x) u_{2}^{*}(y) d \sigma(x, y) .
$$

Proof. First we suppose that $u_{\mu}$ is bounded in $X$. By the conditions (D. 2) and (D. 3) in Definition 1, there exists a sequence $\left(f_{n}\right)$ such that $\left(f_{n}\right)$ converges strongly to $u_{\mu}$ in $D$ as $n \longrightarrow+\infty,\left(f_{n}\right)$ is uniformly bounded and $S_{f_{n}}$ is contained in a fixed neighborhood $N$ of $S_{u_{\mu}}$. We take any fixed element $f$ in $C_{K} \cap D$ such that $S_{f} \subset C S_{u_{\mu}}$. We may assume that the above function $f_{n}$ has the support in $C S_{f}$. Then

$$
\left(f, f_{n}\right)=-2 \iint f_{n}(x) f(y) d \sigma(x, y) .
$$

By Lemma 2 and Lebesgue's bounded convergence theorem, making $n$ tend to infinity, we obtain

$$
\left(f, u_{\mu}\right)=-2 \iint u_{\mu}^{*}(x) f(y) d \sigma(x, y) .
$$

That is,

$$
\int f d \mu^{(1)}=-2 \iint f(x) u_{\mu}^{*}(y) d \sigma(x, y)
$$


Next we shall prove the general case. We may assume that $u_{\mu}$ is nonnegative, because in the general case, $u_{\mu}^{+}$and $u_{\mu}^{-}$are potentials in $D$. Put

$$
u_{\mu, n}(x)=\inf \left(u_{\mu}(x), n\right) \text {. }
$$

Then $u_{\mu, n}$ is contained in $D$ and by the above assertion, we have

$$
\left.\left(u_{\mu, n}, f\right)=-2 \iint f(x) u_{\mu, n}^{*}(y) d \sigma(x, y) .12\right)
$$

Since the sequence $\left(u_{\mu, n}\right)$ converges strongly to $u_{\mu}$ in $D^{13}$ ) and the sequence $\left(u_{\mu, n}(x)\right)$ is non-decreasing, making $n$ tend to infinity, we have

$$
\int f d \mu^{(1)}=\left(u_{\mu}, f\right)=-2 \iint f(x) u_{\mu}^{*}(y) d \sigma(x, y) .
$$

Let's show the second part of our theorem. First we assume that $S_{u_{1}}$ is compact and $u_{2}(x)$ is non-negative. Then we can take a relatively compact open set $\omega_{1}$ and an open set $\omega_{2}$ such that

$$
\overline{\omega_{1}} \cap \overline{\omega_{2}}=\phi, S_{u_{1}} \subset \omega_{1} \text { and } S_{u_{2}} \subset \omega_{2} .
$$

By Lemma 3, we can define a positive measure $\sigma u_{2,1}$ in $\omega_{1}$ such that

$$
\int f d \sigma u_{2,1}=\iint f(x) u_{2}^{*}(y) d \sigma(x, y)
$$

for any $f$ in $C_{K}$ with support in $\omega_{1}$. Let's show that the function $u_{1}^{*}$ is $\sigma u_{2}, 1$-measurable. By the properties of the refinement, there exists a nonincreasing sequence $\left(\omega_{n}\right)$ of open sets contained in $\omega_{1}$ such that $u_{1}^{*}$ is continuous on $C \omega_{n}$ for any $n$ and

$$
\lim _{n \rightarrow \infty} \operatorname{cap}\left(\omega_{n}\right)=0 .{ }^{14)}
$$

We take an open set $\omega_{3}$ such that

$$
\overline{\omega_{2}} \subset \omega_{3} \text { and } \overline{\omega_{1}} \cap \overline{\omega_{3}}=\phi
$$

Let $u_{\mu_{n}}$ be the condensor potential with respect to $\omega_{n}$ and $\omega_{3}$. Then

$$
\int_{\omega_{n}} d \sigma u_{2}, 1 \leq-\frac{1}{2}\left(u_{\mu_{n}}, u_{2}\right) \leq \frac{1}{2}\left\|u_{\mu_{n}}\right\| \cdot\left\|u_{2}\right\|
$$

12) Cf. Proposition 1.

13) Cf. Lemma 4 in [5].

14) For an open set $\omega$, the capacity $\operatorname{cap}(\omega)$ of $\omega$ is defined as follows: $\operatorname{cap}(\omega)=\inf \left\{\|u\|^{2}\right.$; $u(x) \geq 1$ a.e. in $\omega\}, \operatorname{cap}(\omega)=+\infty$ if such elements don't exist. 
Since the sequence $\left(\left\|u_{\mu_{n}}\right\|\right)$ converges to 0 as $n \longrightarrow+\infty, u_{1}^{*}$ is $\sigma u_{2}, 1$-measurable. If $u_{1}^{*}$ is bounded, our conclusion is evident. Put

$$
u_{1, n}^{+}=\inf \left(u_{1}^{+}, n\right), u_{1, n}^{-}=\inf \left(u_{1}^{-}, n\right) .
$$

Then the sequences $\left(u_{1, n}^{+}\right)$and $\left(u_{1, n}^{-}\right)$are non-decreasing and contained in $D$. By the above assertion,

$$
\left(u_{1, n}^{+}, u_{2}\right)=-2 \iint u_{1, n}^{+*}(x) u_{2}^{*}(y) d \sigma(x, y)
$$

and

$$
\left(u_{\overline{1}, n}^{-}, u_{2}\right)=-2 \iint u_{i_{n}, n}^{*}(x) u_{2}^{*}(y) d \sigma(x, y) .
$$

Making $n$ tend to infinity, we obtain

$$
\left(u_{1}^{+}, u_{2}\right)=-2 \iint u_{1}^{+*}(x) u_{2}^{*}(y) d \sigma(x, y) \text { and }\left(u_{1}^{-}, u_{2}\right)=-2 \iint u_{1}^{-*}(x) u_{2}^{*}(y) d \sigma(x, y) .
$$

That is, we have

$$
\left(u_{1}, u_{2}\right)=-2 \iint u_{1}^{*}(x) u_{2}^{*}(y) d \sigma(x, y) .
$$

In the case that $u_{2}$ is general, by the above assertion, we have

$$
\begin{aligned}
& \left(u_{1}, u_{2}\right)=\left(u_{1}, u_{2}^{+}\right)-\left(u_{1}, u_{2}^{-}\right) \\
= & -2 \iint u_{1}^{*}(x) u_{2}^{+*}(y) d \sigma(x, y)+2 \iint u_{1}^{*}(x) u_{2}^{-*}(y) d \sigma(x, y) \\
= & -2 \iint u_{1}^{*}(x) u_{2}^{*}(y) d \sigma(x, y) .
\end{aligned}
$$

Thus we prove the case that $S_{u_{1}}$ is compact. We shall prove the case that $S_{u_{1}}$ is general. Similarly as the above, we may assume that $u_{1}$ and $u_{2}$ are non-negative. We take a non-decreasing net $\left(\omega_{\alpha}\right)_{\alpha \in I}$ of relatively compact open sets tending to $C S_{u_{2}}$. We put $F_{\alpha}=C \omega_{\alpha}$. Let $u_{1, \alpha}^{\prime}$ be the projection of $u_{1}$ to $D_{F_{\alpha}}^{(1)}$, where

$$
D_{F_{\alpha}}^{(1)}=\left\{u_{\mu}: \text { a potential in } D, S_{\mu} \subset F_{\alpha}\right\} \text {. }
$$

Then $u_{1, \alpha}^{\prime}$ is non-negative. ${ }^{15)}$ Furthermore we put

15) Similarly as in [2], p. 214, we obtain the following result: $u^{*}(x) \geq 0$ ppp on the spectrum of $u$ implies $u \geq 0$. Cf. [5]. 


$$
u_{1, \alpha}=u_{1}-u_{1, \alpha}^{\prime}
$$

By the above assertion,

$$
\left(u_{1, \alpha}, u_{2}\right)=-2 \iint u_{1, \alpha}^{*}(x) u_{2}^{*}(y) d \sigma(x, y) .
$$

The net $\left(u_{1, \alpha}^{\prime}\right)$ tends to 0 , and hence the net $\left(u_{1, \alpha}\right)$ tends strongly to $u_{1}$ in $D$. Hence we can choose a subsequence $\left(u_{1, \alpha_{n}}\right)$ of $\left(u_{1, \alpha}\right)$ such that $\left(u_{1, \alpha_{n}}\right)$ converges strongly to $u_{1}$. By Fatou's lemma, we have

$$
\begin{aligned}
& \iint u_{1}^{*}(x) u_{2}^{*}(y) d \sigma(x, y) \leq \frac{\lim }{n \rightarrow \infty} \iint u_{1, \alpha_{n}}^{*}(x) u_{2}^{*}(y) d \sigma(x, y) \\
= & \frac{\lim _{n \rightarrow \infty}}{n \rightarrow \frac{1}{2}}\left(u_{1, \alpha_{n}}, u_{2}\right)=-\frac{1}{2}\left(u_{1}, u_{2}\right) .
\end{aligned}
$$

On the other hand, since $u_{1}^{*}(x)-u_{1, \alpha}^{*}(x) \geq 0 p p p$ in $X$ for any $\alpha \in I$,

$$
\iint u_{1}^{*}(x) u_{2}^{*}(y) d \sigma(x, y) \geq \iint u_{1, \alpha}^{*}(x) u_{2}^{*}(y) d \sigma(x, y) .
$$

Consequently we obtain

$$
\left(u_{1}, u_{2}\right)=-2 \iint u_{1}^{*}(x) u_{2}^{*}(y) d \sigma(x, y)
$$

This completes the proof.

Applying this theorem, we obtain the following corollary.

Let $F$ be a closed set in the product space $X \times X$. The $x$-section $F_{x}$ of $F$ means the projection $\{x\} \times X \cap F$ to $X$, and for an arbitrary subset $A$ of $X$, the $A$-section $F_{A}$ means the union $\underset{x \in A}{\cup} F_{x}$.

Corollary 1. Let $D$ be a Dirichlet space on $X$, and let $\sigma$ be the singular measure of $D$. Given a symmetric closed set $F$ in $X \times X$ containing the diagonal. set $\delta$ of $X \times X$, the following two conditions are equivalent.

(1. 1) For any pure potential $u_{\mu}$ in $D$ and any open set $\omega$ contained in $C S_{\mu}$, let $u_{\mu^{\prime}}$, be the balayaged potential of $u_{\mu}$ to $\omega$. Then

$$
S_{\mu^{\prime}} \subset F_{C \omega} \cap \bar{\omega} \text {. }
$$

$$
S_{\sigma} \subset F \text {. }
$$

In the preceding paper [4], we proved this result in the case that $F$ is regular, i.e., $F_{x}$ is compact for any $x \in X$ and the point-to-set map: $x \longrightarrow F_{x}$ 
is continuous. Let's prove this corollary. First we shall prove the implication (1. 1) $\Rightarrow(1.2)$. Suppose that $S_{\sigma} \not \subset F$. Then there exist two functions $f_{1}$ and $f_{2}$ in $C_{K}^{+} \cap D$ such that

$$
S_{f_{1}} \cap F_{S_{f_{2}}}=\phi, \quad S_{f_{2}} \cap F_{S_{f_{1}}}=\phi
$$

and

$$
\left.\iint f_{1}(x) f_{2}(y) d \sigma(x, y)>0.16\right)
$$

Hence there exists a pure potential $u_{\mu}$ in $D$ such that $S_{\mu} \subset S_{f_{1}}$ and

$$
\iint\left(u_{\mu}^{*}(x)-u_{\mu^{\prime}}^{*}(x)\right) f_{2}(y) d \sigma(x, y)>0,
$$

where $u_{\mu^{\prime}}$ is the balayaged potential of $u_{\mu}$ to $C S_{f_{1}}$. On the other hand, since

$$
S_{\left(u_{\mu}-u_{\mu^{\prime}}\right)} \cap S_{f_{2}}=\phi
$$

we have

$$
2 \iint\left(u_{\mu}^{*}(x)-u_{\mu^{\prime}}^{*}(x)\right) f_{2}(y) d \sigma(x, y)=\int f_{2}(x) d \mu^{\prime}(x)=0,
$$

because

$$
S_{\mu^{\prime}} \subset F_{S_{f_{1}}}
$$

by our assumption. This is a contradiction. The proof of the implication (1. 2) $\Rightarrow(1.1)$ is evident by the fact that $u_{\mu}(x)-u_{\mu^{\prime}}(x)=0$ a.e. in $\omega$ and Theorem 1. This completes the proof.

In order to characterize the absolute continuity of balayaged measures, first we give the following definition.

Definition 5. Let $\sigma$ be the singular measure of a Dirichlet space $D$. We say that the projection of $\sigma$ to $X$ is absolutely continuous for $\xi$ if for any compact set $K$ in $X$, the positive measure $\sigma_{K}$ in $C K$ is absolutely continuous for $\xi$.

Remark. If $\sigma$ is absolutely continuous for $\xi \times \xi$, the projection of $\sigma$ to

16) Cf. [4], Lemma 6. 
$X$ is absolutely continuous for $\xi$. But the converse is not valid. We can easily construct a counter example.

Another corollary of Theorem 1 is the following

Corollary 2. Let $D$ be a Dirichlet space on $X$ and $\sigma$ be the singular measure of $D$. The following two conditions are equivalent.

(2. 1) For any pure potential $u_{\mu}$ in $D$ and any open set $\omega$ contained in $C S_{\mu}$, let $u_{\mu^{\prime}}$ be the balayaged potential of $u_{\mu}$ to $\dot{\omega}$. Then the restriction of $\mu^{\prime}$ to $\omega$ is absalutely continuous for $\xi$.

(2. 2) The projection of $\sigma$ to $X$ is absolutely continuous for $\xi$.

Proof. First we shall prove the implication (2.1) $\Rightarrow(2.2)$. For a compact set $K$ in $X$, it is sufficient to prove that the positive measure $\sigma_{K}$ is absolutely continuous for $\xi$ in any open set $\omega$ such that $\bar{\omega} \subset C K$. We take another open set $\omega_{1}$ in $X$ such that

$$
K \subset \omega_{1}, \overline{\omega_{1}} \cap \bar{\omega}=\phi .
$$

Let $u_{\mu_{1}-\mu_{0}}$ be the condensor potential with respect to $\omega_{1}$ and $\omega$. By Theorem 1 , for any $f$ in $C_{K}^{+}$with support in $\omega$, we have

$$
\int f d \sigma_{K} \leq \iint f(x) u_{\mu_{1}-\mu_{0}}^{*}(y) d \sigma(x, y)=\frac{1}{2} \int f d \mu_{0} .
$$

That is, the inequality $\sigma_{K} \leq \frac{1}{2} \mu_{0}$ holds in $\omega$. Since $u_{\mu_{1}}$ is contained in $D$ and $\mu_{0}$ is the balayaged measure of $\mu_{1}$ to $\omega$, we obtain that $\sigma_{K}$ is absolutely continuous for $\xi$ in $\omega$.

Next we shall prove the converse. First suppose that $C \omega$ is compact in $X$. By Theorem 1, the restriction $\mu^{\prime(1)}$ of $\mu^{\prime}$ to $\omega$ satisfies the following:

$$
\int f d \mu^{\prime(1)}=2 \iint f(x)\left(u_{\mu}^{*}(y)-u_{\mu^{\prime}}^{*}(y)\right) d \sigma(x, y)
$$

for any $f$ in $C_{K}$ with support in $\omega$. Hence it is evident that the condition (2. 1) is satisfied if $u_{\mu}^{*}(x)-u_{\mu^{\prime}}^{*}(x)$ is bounded. In the general case, we put

$$
u_{n}(x)=\inf \left(u_{\mu}(x)-u_{\mu^{\prime}}(x), n\right) .
$$

Then $u_{n}$ is in $D$. By our assumption, for any compact set $K$ in $X$ such that $\xi(K)=0$ and $K \subset \omega$, 


$$
\int_{K} \int u_{n}^{*}(x) d \sigma(x, y)=0
$$

Making $n$ tend to infinity, we obtain

$$
\int_{K} \int\left(u_{\mu}^{*}(x)-\mu_{\mu^{\prime}}^{*}(x)\right) d \sigma(x, y)=0,
$$

and hence $\mu^{\prime}(K)=0$. That is, $\mu^{\prime(1)}$ is absolutely continuous for $\xi$. Next we shall prove the case that $\omega$ is general. We take a decreasing net $\left(\omega_{\alpha}\right)_{\alpha \in I}$ of open sets such that $C \omega_{\alpha}$ is compact in $X$ for any $\alpha \in I$ and it tend to $\omega$. Let $u_{\mu_{\alpha}^{\prime}}$ be the balayaged potential of $u_{\mu}$ to $\omega_{\alpha}$. Then the positive measure $\mu_{\alpha}^{\prime(1)}$ is absolutely continuous for $\xi$. Since the net $\left(u_{\mu_{\alpha}^{\prime}}\right)$ is nondecreasing and converges strongly to $u_{\mu^{\prime}}$, there exists a subsequence $\left(u_{\mu_{\alpha_{n}}^{\prime}}\right)$ of $\left(u_{\mu_{\alpha}^{\prime}}\right)$ which is non-decreasing and converges strongly to $u_{\mu^{\prime}}$ as $n \longrightarrow+\infty$. Similarly as the above calculation, we obtain that $\mu^{\prime(1)}$ is absolutely continuous for $\xi$.

This completes the proof.

\section{Second main theorems}

In this section, first we shall examine some properties of equilibrium measures and equilibrium potentials in a Dirichlet space. ${ }^{17)}$ We shall prove the following lemmas.

Lemma 4. Let $D$ be a Dirichlet space on $X$. For an open set $\omega$ in $X$, the equilibrium potential $u_{\nu}$ of $\omega$ exists in $D$ if cap $(\omega)<+\infty$.

Proof. By the definition of the capacity, the set

$$
E_{\omega}=\{u \in D ; u(x) \geq 1 \text { a.e. in } \omega\}
$$

is non-empty and closed convex subset of $D$. Similarly as Beurling \& Deny [2], a unique element whose norm is minimum in $E$ is the equilibrium potential of $\omega$.

Lemma 5. Let $D$ be a Dirichlet space on $X$. For two open sets $\omega_{1}$ and $\omega_{2}$

17) Let $D$ be a Dirichlet space on $X$. Beurling and Deny [2] showed that for any relatively compact open set $\omega$, there exists a pure potential $u_{\nu}$ in $D$ such that $0 \leq u_{\nu} \leq 1, u_{\nu}=1$ a.e. in $\omega$ and $S_{\nu} \subset \bar{\omega}$. This potential $u_{\nu}$ is called the equilibrium potential of $\omega$ and this positive measure $\nu$ is called the equilibrium measure of $\omega$. 
in $X$ such that $\omega_{1} \subset \omega_{2}$ and $\operatorname{cap}\left(\omega_{2}\right)<+\infty$, let $u_{\nu_{1}}$ and $u_{\nu_{2}}$ be the equilibrium potentials of $\omega_{1}$ and $\omega_{2}$, respectively. Then, for any Borel set $A$ contained in $\omega_{1}$,

$$
\nu_{1}(A) \geq \nu_{2}(A) \text {. }
$$

Proof. It is sufficient to prove that for any $f$ in $C_{K}^{+} \cap D$ with support in $\omega_{1}$,

$$
\int f d \nu_{1} \geq \int f d \nu_{2}
$$

because $C_{K}^{+}\left(\omega_{1}\right) \cap D$ is dense in $C_{K}^{+}\left(\omega_{1}\right){ }^{18)} \quad$ Using the domination theorem, we obtain that

$$
u_{\mu_{2}} \geq u_{\mu_{1}} \text { and } S_{\left(u_{\mu_{2}}-u_{\mu_{1}}\right)} \subset C \omega_{1}
$$

Then by Theorem 1, we have

$$
\int f d \mu_{1}-\int f d \mu_{2}=2 \iint f(x)\left(u_{\mu_{2}}^{*}(y)-u_{\mu_{1}}^{*}(y)\right) d \sigma(x, y) \geq 0 .
$$

This completes the proof.

By Lemma 4, for any open set $\omega$ in $X$, there exists a positive measure $\nu$ supported by $\bar{\omega}$ such that for any net $\left(\omega_{\alpha}\right)$ of relatively compact open sets contained in $\omega$ tending to $\omega$, the equilibrium measure $\nu_{\alpha}$ of $\omega_{\alpha}$ converges vaguely to $\nu$. We say that this positive measure $\nu$ is the equilibrium measure of $\omega$. Similarly as the above, we obtain the following

Lemma $5^{\prime}$. Let $D$ be a Dirichlet space on $X$. For two open sets $\omega_{1}$ and $\omega_{2}$ such that $\omega_{1} \subset \omega_{2}\left(c a p\left(\omega_{2}\right)\right.$ is finite or not $)$, let $\nu_{i}$ be the equilibrium measure of $\omega_{i}$ for $i=1,2$. Then for any Borel set $A$ contained in $\omega_{1}$,

$$
\nu_{1}(A) \geq \nu_{2}(A) .
$$

This follows immediately from the above lemma. By the above two lemmas, we obtain the following corollary.

Corollary 3. Let $D$ be a Dirichlet space on $X$. Suppose that for any relatively compact open set $\omega$ in $X$, the equilibrium measure $\nu$ of $\omega$ is absolutely continuous for $\xi$. Then, for any open set $\omega$ in $X$, the equilibrium measure $\nu$ of $\omega$

18) Because the closure of $C_{K}\left(\omega_{1}\right) \cap D$ by the norm of $D$ is a Dirichlet space on $\omega_{1}$. Cf. [5]. 
is absolutely continuous for $\xi$. Especially the equilibrium measure of $X$ is absolutely continuous for $\xi$.

Similarly as in Theorem 1, we obtain the following theorem.

Theorem 2. Let $D$ be a Dirichlet space on $X$, and let $\nu$, $\sigma$ be the equilibrium measure of $X$, the singular measure of $D$, respectively. For an open set $\omega$ in $X$ with cap $(\omega)<+\infty$, let $\mu$ be the equilibrium measure of $\omega$ and $\mu^{(1)}$ be the restriction of $\mu$ to $\omega$. Then

$$
\int f d \mu^{(1)}=2 \iint f(x)\left(u_{\mu}^{*}(x)-u_{\mu}^{*}(y)\right) d \sigma(x, y)+\int f d \nu
$$

for any $f$ in $C_{K}$ with support in $\omega$. Furthermore, for any couple $u_{1}$ and $u_{2}$ in $D$ such that $u_{2}(x)=c$ a.e. in some neighborhood of $S_{u_{1}}$,

$$
\left(u_{1}, u_{2}\right)=c \int u_{1}^{*}(x) d \nu(x)+2 \iint u_{1}^{*}(x)\left(u_{2}^{*}(x)-u_{2}^{*}(y)\right) d \sigma(x, y),
$$

where $c$ is constant.

In order to prove this theorem, we need the following lemma.

Lemma 6. Let $D$ be a Dirichlet space on $X$. Given a relatively compact open set $\omega$ in $X$, let $u_{\mu}$ be the equilibrium potential of $\omega$. Then there exist unrefinement $u_{\mu}^{*}$ of $u_{\mu}$ such that the equality $u_{\mu}^{*}(x)=1$ holds everywhere in $\omega$.

Proof. It is sufficient to prove that for any open set $\omega_{1}$ such that $\overline{\omega_{1}} \subset \omega$, the equality $u_{\mu}^{*}(x)=1$ holds everywhere in $\omega_{1}$. By Lemma 1 , there exists a sequence $\left(f_{n}\right)$ in $C_{K} \cap D$ such that $\left(f_{n}\right)$ converges strongly to $u_{\mu}$ as $n \longrightarrow+\infty, 0 \leq f_{n} \leq 1$ and $f_{n}(x)=1$ in $\omega_{1}$ for any $n$. We may assume that

$$
\sum_{n=1}^{\infty} 4^{n}\left\|f_{n+1}-f_{n}\right\|^{2}<+\infty
$$

By the definition of the refinement, the sequence $\left(f_{n}\right)$ is uniformly convergent to $u_{\mu}^{*}$ in $C E_{k}$, where

$$
E_{k}=\bigcup_{n=k}^{\infty} E_{n}^{\prime}=\bigcup_{n=k}^{\infty}\left\{x \in X ;\left|f_{n+1}(x)-f_{n}(x)\right|>1 / 2^{n}\right\}
$$

for any integer $n$. The inclusion $\omega_{1} \subset C E_{k}$ exists for any integer $n$, and hence we obtain that $u_{\mu}^{*}$ is continuous in $\omega_{1}$ and the equality $u_{\mu}^{*}(x)=1$ holds everywhere in $\omega_{1}$. This completes the proof. 
Remark. The above lemma is valid for any open set $\omega$ with finite capacity.

Proof of Theorem 2. Let $\omega$ be the open set in our theorem. For any $f$ in $C_{K}^{+}$supported in $\omega$, let $\sigma_{f}$ be a positive measure in $C S_{f}$ similarly as in the proof of Theorem 1. By Lemma 1 and Theorem 1, the function $1-u_{\mu}^{*}(x)$ is $\sigma_{f}$-integrable. Let $\left(f_{n}\right)$ be a sequence in $C_{K} \cap D$ such that $\left(f_{n}\right)$ converges strongly to $u_{\mu}$ in $D$ as $n \longrightarrow+\infty, 0 \leq f_{n}(x) \leq 1$ and $f_{n}(x)=1$ in some neighborhood of $S_{f}$ for any $n$. Then by Beurling-Deny's representation theorem, we have

$$
\begin{aligned}
& \left(f_{n}, f\right)=\int f(x) d \nu(x)+\iint(f(x)-f(y))\left(f_{n}(x)-f_{n}(y)\right) d \sigma(x, y) \\
= & \int f(x) d \nu(x)+2 \int f(x)\left(1-f_{n}(y)\right) d \sigma(x, y) .
\end{aligned}
$$

By Lebesgue's bounded convergence theorem, we obtain that

$$
\begin{aligned}
& \int f(x) d \mu(x)=\left(u_{\mu}, f\right) \\
= & \int f(x) d \nu(x)+2 \iint f(x)\left(1-u_{\mu}^{*}(y)\right) d \sigma(x, y) \\
= & \int f(x) d \nu(x)+2 \iint f(x)\left(u_{\mu}^{*}(x)-u_{\mu}^{*}(y)\right) d \sigma(x, y) .
\end{aligned}
$$

From this equality, we obtain the first required equality. Let's prove the second part of our theorem. We may assume that $u_{2}^{*}$ is equal to $c$ everywhere in some neighborhood $\omega$ of $S_{u_{1}}$. Similarly as the proof of Theorem 1 and the proof of the first part of our theorem, we obtain

$$
\left(u_{1}, u_{2}\right)=c \int u_{1}^{*}(x) d \nu(x)+2 \iint u_{1}^{*}(x)\left(u_{2}^{*}(x)-u_{2}^{*}(y)\right) d \sigma(x, y) .
$$

In the above equality, the $\nu$-measurablity of $u_{1}^{*}$ is followed from Lemma 5 . This completes the proof.

As an application of the above theorem, we obtain the following theorem. This result is more precise than in [5].

Theorem 3. Let $D$ be a Dirichlet space on $X$ and $\nu$ be the equilibrium measure of $X$. For a pure potential $u_{\mu}$ in $D$ such that $\int d \mu<+\infty$ and an open set $\omega$ in $X$ such that cap $(C \omega)<+\infty$, let $u_{\mu^{\prime}}$ be the balayaged potential of $u_{\mu}$ to $\omega$. Then 


$$
\int\left(u_{\mu}^{*}(x)-u_{\mu^{\prime}}^{*}(x)\right) d \nu(x)=\int d \mu-\int d \mu^{\prime}
$$

Furthermore, for a non-decreasing net $\left(K_{\alpha}\right)_{\alpha \in I}$ of compact sets in $X$ tending to $X$, let $u_{\mu_{\alpha}^{\prime}}$ be the balayaged potential of $u_{\mu}$ to $\omega_{\alpha}=C K_{\alpha}$. Then the net $\left(\int d \mu_{\alpha}^{\prime}\right)_{\alpha \in I}$ is non-increasing and

$$
\int u_{\mu}^{*}(x) d \nu(x)=\int d \mu-a_{\mu}
$$

where

$$
a_{\mu}=\lim _{\alpha \in I} \int d \mu_{\alpha}^{\prime} .
$$

Before we give the proof of this theorem, we remark the following

Corollary 3. Let the notations be the same as in the above theorem. For any pure potential $u_{\mu}$ in $D$ with $\int d \mu<+\infty$ and any open set $\omega$ in $X$ such that $\operatorname{cap}(C \omega)<+\infty, \int d \mu=\int d \mu^{\prime}\left(\operatorname{res} p . \int d \mu>\int d \mu^{\prime}\right)$ if and only if $\nu=0$ (resp. $\nu$ is everywhere dense in $X$ ).

The proof of this corollary is immediate from the above theorem. This corollary was partially proved in [5].

Proof of Theorem 3. First we shall prove the case that $C_{\omega} \omega$ is compact in $X$. We take a non-decreasing net $\left(\omega_{\alpha}\right)_{\alpha \in I}$ of relatively compact open sets in $X$ such that $\omega_{\alpha} \supset C \omega$ for any $\alpha \in I$ and the net $\left(\omega_{\alpha}\right)$ tends to $X$. Then, for any $\alpha \in I$, we have

$$
\begin{aligned}
& \left(u_{\mu}-u_{\mu^{\prime}}, u_{\mu_{\alpha}}\right)=\int\left(u_{\mu}^{*}(x)-u_{\mu^{\prime}}^{*}(x)\right) d \mu_{\alpha}(x) \\
= & \int\left(u_{\mu}^{*}(x)-u_{\mu^{\prime}}^{*}(x)\right) d \nu(x)+2 \iint\left(u_{\mu}^{*}(x)-u_{\mu^{\prime}}^{*}(x)\right)\left(u_{\mu_{\alpha}}^{*}(x)-u_{\mu_{\alpha}}^{*}(y)\right) d \sigma(x, y) \\
= & \int\left(u_{\mu}^{*}(x)-u_{\mu^{\prime}}^{*}(x)\right) d \nu(x)+2 \iint\left(u_{\mu}^{*}(x)-u_{\mu^{\prime}}^{*}(x)\right)\left(1-u_{\mu_{\alpha}}^{*}(y)\right) d \sigma(x, y) .
\end{aligned}
$$

Since the net $\left(1-u_{\mu_{\alpha}}^{*}\right)_{\alpha \in I}$ is non-increasing and tends to 0 in $X$, the second part of the last hand converges non-increasingly to 0 . Hence we have

$$
\lim _{\alpha \in I}\left(u_{\mu}-u_{\mu^{\prime}}, u_{\mu_{\alpha}}\right)=\int\left(u_{\mu}^{*}(x)-u_{\mu^{\prime}}^{*}(x)\right) d \nu(x) .
$$


On the other hand, the net $\left(u_{\mu_{\alpha}}\right)_{\alpha \in I}$ tending non-decreasingly to 1 in $X$, we obtain that

$$
\lim _{\alpha \in I}\left(u_{\mu}, u_{\mu_{\alpha}}\right)=\int d \mu, \quad \lim _{\alpha \in I}\left(u_{\mu^{\prime}}, u_{\mu_{\alpha}}\right)=\int d \mu^{\prime}
$$

That is,

$$
\int\left(u_{\mu}^{*}(x)-u_{\mu^{\prime}}^{*}(x)\right) d \nu(x)=\int d \mu-\int d \mu^{\prime}
$$

Next we shall show the case that $C_{\omega}$ is general. We take a decreasing net $\left(\omega_{\alpha}\right)_{\alpha \in I}$ of open sets such that $C \omega_{\alpha}$ is compact and $\omega_{\alpha} \supset \omega$ for any $\alpha \in I$, and that the net tends to $\omega$. Let $\nu^{\prime}$ be the restriction of $\nu$ to some fixed open set containing $C \omega$ with finite capacity. By Lemma 5, a potential $u_{\nu^{\prime}}$ exists in $D$. Hence

$$
\begin{aligned}
& \int\left(u_{\mu}^{*}(x)-u_{\mu_{\alpha}^{\prime}}^{*}(x)\right) d \nu(x)=\int\left(u_{\mu}^{*}(x)-u_{\mu_{\alpha}^{\prime}}^{*}(x)\right) d \nu^{\prime}(x) \\
= & \left(u_{\mu}-u_{\mu_{\alpha}^{\prime}}, u_{\nu^{\prime}}\right) \longrightarrow\left(u_{\mu}-u_{\mu^{\prime}}, u_{\nu^{\prime}}\right) \\
= & \left(u_{\mu}^{*}(x)-u_{\mu^{\prime}}^{*}(x)\right) d \nu(x),
\end{aligned}
$$

because the net $\left(u_{\mu}-u_{\mu_{\alpha}^{\prime}}\right)_{\alpha \in I}$ converges strongly to $u_{\mu}-u_{\mu^{\prime}}$, in $D$, where $\mu_{\alpha}^{\prime}$ is the balayaged measure of $\mu$ to $\omega_{\alpha}$. On the other hand, similarly as the proof of theorem 1 in [5],

$$
\lim _{\alpha \in I} \int d \mu_{\alpha}^{\prime}=\int d \mu^{\prime}
$$

Thus the first part of our theorem is proved and the second part can be obtained by the usual limiting process. This completes the proof.

Evidently we know that $a_{\mu}$ vanishes for any pure potential $u_{\mu}$ in $D$ when $X$ is of finite capacity. But we don't know the condition which $a_{\mu}$ vanishes. Finally we remark that similar theorems as Theorem 1 and Theorem 3 hold for a condensor measure.

\section{Special Dirichlet spaces}

First, according to Beurling and Deny [2], we define a special Dirichlet space.

Definition 4. A Dirichlet space $D=D(X ; \xi)$ is said to be special if $X$ 
is a locally compact abelian group, $\xi$ is the Haar measure of $X$ and the following condition is satisfied:

(D. 4) For any $u$ in $D$ and any $x$ in $X$, the function $U_{x} u$ is in $D$ and $\left\|U_{x} u\right\|=\|u\|$, where $U_{x} u$ is the function obtained from $u$ by the translation $x$ (i.e., $U_{x} u(y)=u(y-x)$ ).

In the case that $D$ is a special Dirichlet space on $X$, Proposition 1 reads as follows:

Proposition 3. Let $D$ be a special Dirichlet space on $X$. Then there exists a positive constant $c$, a local form $N(\cdot, \cdot)$ on $C_{K} \cap D$ and a positive symmetric measure $\sigma^{\prime}$ in $X-\{0\}$ such that

$$
(f, g)=c \int f g d \xi+N(f, g)+\iint(f(x+y)-f(x))(g(x+y)-g(x)) d \sigma^{\prime}(y) d \xi(x)
$$

for any pair $f$ and $g$ in $C_{K} \cap D$. The above representation is unique.

Proof. By Proposition 1, there exist a positive measure $\nu$ in $X$ and a positive symmetric measure $\sigma$ in $X \times X-\delta$ such that

$$
(f, g)=\int f g d \nu+N(f, g)+\iint(f(x)-f(y))(g(x)-g(y)) d \sigma(x, y)
$$

for any pair $f$ and $g$ in $C_{K} \cap D$. We take an increasing net $\left(K_{\alpha}\right)$ of compact sets in $X$ which tends to $X$ and an increasing net $\left(g_{\alpha}\right)$ of $C_{K} \cap D$ such that $0 \leq g_{\alpha}(x) \leq 1, g_{\alpha}(x)=1$ on $K_{\alpha}$ for any $\alpha \in I$ and the net $\left(g_{\alpha}\right)$ tends to 1 in $X$. We know the existence of this function $g_{\alpha}$ by the condition (D. 2) and (D. 3). For any $f$ in $C_{K} \cap D$ and any $x$ in $X$,

$$
\lim _{\alpha \in I}\left(f, g_{\alpha}\right)=\int f d \nu, \quad \lim _{\alpha \in I}\left(U_{x} f, U_{x} g_{\alpha}\right)=\int U_{x} f d \nu,
$$

and hence

$$
\int f d \nu=\int U_{x} f d \nu
$$

Consequently $d \nu=c d \xi$, where $c$ is a non-negative constant. Next we shall examine the singular measure $\sigma$ of $D$. For any $f$ and $g$ in $C_{K}^{+}$such that the support $S_{f * g}$ of the convolution $f * g$ doesn't contain the origin 0 of $X$, the transformation

$$
f * g \longrightarrow \iint f(x) g(y) d \sigma(x, y)
$$


is positive linear. In fact, suppose that $f_{1} * g_{1} \leq f_{2} * g_{2}$. For any $h$ in $C_{K}^{+}$ such that $S_{h} \cap S_{f_{2^{*}} g_{2}}=\phi$,

$$
\begin{aligned}
\iint f_{1}(x) g_{1} * h(y) d \sigma(x, y) & =\iint f_{1} * g_{1}(x) h(y) d \sigma(x, y) \\
\leq \iint f_{2} * g_{2}(x) h(y) d \sigma(x, y) & =\iint f_{2}(x) g_{2} * h(y) d \sigma(x, y) .
\end{aligned}
$$

Making $h$ vaguely tend to the unit measure $\varepsilon$ at 0 , we obtain

$$
\iint f_{1}(x) g_{1}(y) d \sigma(x, y) \leq \iint f_{2}(x) g_{2}(y) d \sigma(x, y) \text {. }
$$

The well-definedness of the above transformation is evidently followed by our assumption, i.e.,

$$
\iint f(x) g(y) d \sigma(x, y)=\iint f\left(x+x_{0}\right) g\left(y+x_{0}\right) d \sigma(x, y)
$$

for any $x_{0}$ in $X$. Since the totality of such functions $f * g$ is dense in $C_{K}^{+}(X-\{0\})$, there exists a positive measure $\sigma^{\prime}$ in $X-\{0\}$ such that

$$
\int f * g(x) d \sigma^{\prime}(x)=\iint f(x) g(y) d \sigma(x, y)
$$

for any pair $f$ and $g$ in $C_{K}^{+}$such that $S_{f} \cap S_{g}=\phi$. The symmetricity of $\sigma^{\prime}$ follows from the simmetricity of $\sigma$. Consequently

$$
\iint f(x+y) g(x) d \sigma^{\prime}(y) d \xi(x)=\iint f(x) g(y) d \sigma(x, y) .
$$

The uniqueness of the singular measure of $D$ follows from the equality

$$
\begin{aligned}
& \iint(f(x+y)-f(x))(g(x+y)-g(x)) d \sigma^{\prime}(y) d \xi(x) \\
= & \iint(f(x)-f(y))(g(x)-g(y)) d \sigma(x, y)
\end{aligned}
$$

for any pair $f$ and $g$ in $C_{K} \cap D$, and hence the proof is completed.

In this case, we call the above positive measure $\sigma^{\prime}$ the singular measure of $D$. Furthermore the local form $N(\cdot, \cdot)$ satisfies the following condition: $N(f, g)=N\left(U_{x} f, U_{x} g\right)$ for any pair $f, g$ in $C_{K} \cap D$ and any $x$ in $X$. Hence the above proof is one of Levy-Khinchine's theorem. ${ }^{19}$ Then we obtain the following corollary.

19) Cf. [2], [3], and [4]. 
Corollary 4. Let $D$ be a special Dirichlet space on $X$. The above positive constant $c$ doesn't vanish if and only if $D \subset L^{2}$ and the mapping: $f \longrightarrow f$ on $D$ into $L^{2}$ is continuous.

The proof is evident by the above proposition. As another application of the above proposition, we obtain the following

Theorem 4. Let $D$ be a special Dirichlet space on $X$, and let $\sigma$ be the singular measure of $D$. For any pure potential $u_{\mu}$ in $D$ and any open set $\omega$ contained in $C S_{\mu}$, let $u_{\mu \prime}$ be the balayaged potential of $u_{\mu}$ to $\omega$, and let $\mu^{\prime(1)}$ be the restriction of $\mu^{\prime}$ to $\omega$. Then $\mu^{\prime(1)}$ is absolutely continuous for $\xi$.

Proof. By Theorem 1,

$$
\begin{aligned}
& \int f d \mu^{\prime}=-\left(u_{\mu}-u_{\mu^{\prime}}, f\right) \\
= & 2 \iint\left(u_{\mu}^{*}(x+y)-u_{\mu^{\prime}}^{*}(x+y)\right) f(x) d \sigma(y) d \xi(x)
\end{aligned}
$$

for any $f$ in $C_{K} \cap D$ with support in $\omega$. Now the function

$$
f_{\mu, \omega}(x)=2 \int\left(u_{\mu}^{*}(x+y)-u_{\mu^{\prime}}^{*}(x+y)\right) d \sigma(y)
$$

is a locally summable function in $\omega$, and hence $\mu^{\prime(1)}$ is absolutely continuous for $\xi$. This completes the proof.

Similarly as in Theorem 4, we obtain that $\mu^{\prime(1)}$ is a function of class $C^{\infty}$ in $\omega$ if and only if $\sigma$ is a function of class $C^{\infty}$ in $R^{n}-\{0\}$, where $D$ is a special Dirichlet space on the $n$-dimensional Euclidean space $R^{n}(n \geq 1)$. (Cf. [7])

\section{REFERENCES}

[1] A. Beurling \& J. Deny: Espaces de Dirichlet I, Le case élémentaire, Acta Math., 99 (1958), 103-124.

[ 2 ] -: Dirichlet spaces, Proc. Nat. Acad. Sc. U.S.A., 45 (1959), 208-215.

[ 3 ] J. Deny: Sur les espaces de Dirichlet, Sém, théorie du potentiel, Paris, 1957.

[4] M. Itô: Characterizations of supports of balayaged measures, Nagoya Math. J., 28 (1966), 203-230.

[ 5 ] — : On total masses of balayaged measures, Nagoya Math. J., 30 (1967), 263-278.

[6] —-: Condensor principle and the unit contraction, Nagoya Math. J., 30 (1967), $9-28$. 
[ 7 ] H. Wallin: Regularity properties of the equilibrium distribution, Ann. Inst. Fourier, 15 (1965), 71-90.

\section{Mathematical Institute \\ Nagoya University}

